\title{
Analysis of root crop preparation system
}

\author{
Aleksey Brusenkov ${ }^{1}$, Vasiliy Kapustin ${ }^{1}$, Vladimir Nemtinov ${ }^{1 *}$ and Yulia Nemtinova ${ }^{1,2}$ \\ ${ }^{1}$ Tambov State Technical University, 392000, Sovetskaya st., 106, Tambov, Russia \\ ${ }^{2}$ Tambov State Tambov State University named after G.R. Derzhavin, 392000, Internatsionalnaya st., \\ 33, Tambov, Russia
}

\begin{abstract}
In recent years, the technical level of agricultural production has increased significantly, successfully introduced new technological techniques, means of mechanization and automation of production in crop and livestock. At the same time, further progress in this direction is impossible without systematically organized work on the development and implementation of modern automatic control systems for various processes. Works in this direction are based primarily on a detailed study of the properties of various objects of agricultural production, as the basis for the analysis and synthesis of effective automatic control systems is mathematical modeling of real processes and devices. Recently, there has been a transition from the modeling of individual devices to the modeling of complex technological schemes, which is largely due to a significant increase in the complexity and dimension of the problems to be solved. Therefore, this approach allows you to set and solve optimal control problems not only for individual devices, but for entire technological complexes, which in turn will provide a significant economic effect and will be widely used in solving many engineering problems.
\end{abstract}

\section{Introduction}

One of the most time-consuming processes on livestock farms of various forms of ownership is the preparation of feed for feeding. The task of their preparation for feeding is to ensure that as a result of mechanical (grinding, mixing) or heat treatment (steaming) to increase the content of chemical elements and digestibility of animals consumed feed mixture. This principle is the basis of both the design of machines and equipment, and the entire mode of technological process of preparation of feed on livestock farms and complexes. Therefore, increasing the efficient use of nutritional value with the introduction of intensive technologies of preparation of complete feed mixtures with the use of root crops based on high-efficiency equipment is one of the most important tasks in animal husbandry $[1,2,3]$. Many different components are used in animal feeding diets, and therefore the process of preparing root crops for fodder for agricultural animals is currently not fully worked out, since it is quite difficult to mechanize it with one machine. For mechanization of production processes on livestock farms and complexes use various

\footnotetext{
*Corresponding author: nemninov@mail.gaps.tstu.ru
} 
machines and equipment that are used for the preparation, transportation and distribution of feed and are used both individually and as part of the production lines (PTL) $[4,5,6]$.

Technological lines of preparation of root crops provide performance of operations of loading, delivery, unloading, accumulation (storage), giving, clearing or washing, grinding and the metered giving on the line of mixing [7, 8, 9, 10]. For the successful management of technological processes performed by the working bodies of forage machines, it is no longer enough to know the individual qualitative aspects of the process. Therefore, the systematic approach and methods of mathematical modeling, which are the initial basis for the study and design of modern machines, are increasingly being used [11, 12, 13].

A deep study of the mechanism of technological processes of machines for the purpose of their mathematical description for the subsequent optimization of the main parameters is a rather difficult task. The fact is that in addition to comprehensive information about the influence of a large number of different factors on the process under study, it is necessary to have accurate methods of their theoretical generalization. Therefore, the use of mathematical modeling methods in engineering and scientific research is a significant step forward on the path of technological progress and scientific knowledge [14, 15].

Mathematical modeling is the study of the behavior of an object in certain conditions by compiling and solving equations of its mathematical model. From a large number of definitions, in our opinion, the following is optimal: "a Mathematical model is a specific information object in the form of a system of mathematical relations, which are an approximate formal description of the properties, characteristics and relationships of the original object of arbitrary nature, which are essential for the problem solved by the subject (person)" [16, 17]. Thus, each mathematical model is a structure, the components of which are: the subject (person); the problem solved by the subject; the object-the original (fragment of reality) and the language of formal description of the model by means of mathematical symbolism.

If the model is an idealization of a real object, when the determining and discarded the secondary (specific tasks) traits or characteristics. It is quite obvious that each material object can be compared with many different models that differ from one another both in their structure and the degree of detail of the properties of the object in question, and the way they are obtained (description of the data table, graph, formula, system of equations, algorithm, and the like).

A well-constructed mathematical model is generally more accessible to study than a real object. In addition, it has a remarkable property: its study gives some new knowledge about the original object. However, each model has certain limitations and assumptions associated with the problem to be solved and the properties of the original object. The quality of the resulting models can not be estimated either by structure or form. The only criterion for such an assessment can only be the reliability of the predictions of the behavior of a real object obtained on the model. Moreover, one of the possible models may be more adequate than others in a certain range of variable factors, but less accurate in another range. In this case, you can choose a less accurate model, if it is adequate in the critical (optimal) area.

\section{Procedure for analyzing engineering solutions for complex systems}

A large number of factors affecting the efficiency of technological processes in the production of livestock products, their complex nature, the difference in the evaluation criteria of significance, complex relationships, make it necessary to use a systematic approach to their production. A biotechnology system that consists of the set technological processes, ranging from preparation of feed rations, including washing and chopping root 
crops to produce animal products with the help of machines and equipment to ensure their function is considered for the first time.

Figure 1 shows the technological scheme of BTS delivery of root crops to the point of preparation for their washing, grinding and subsequent delivery to cattle in accordance with the diet of its feeding. It includes a trailer feeder-dispenser 1 with an inclined conveyor 2, a sink-chopper 3 and a sump 4.
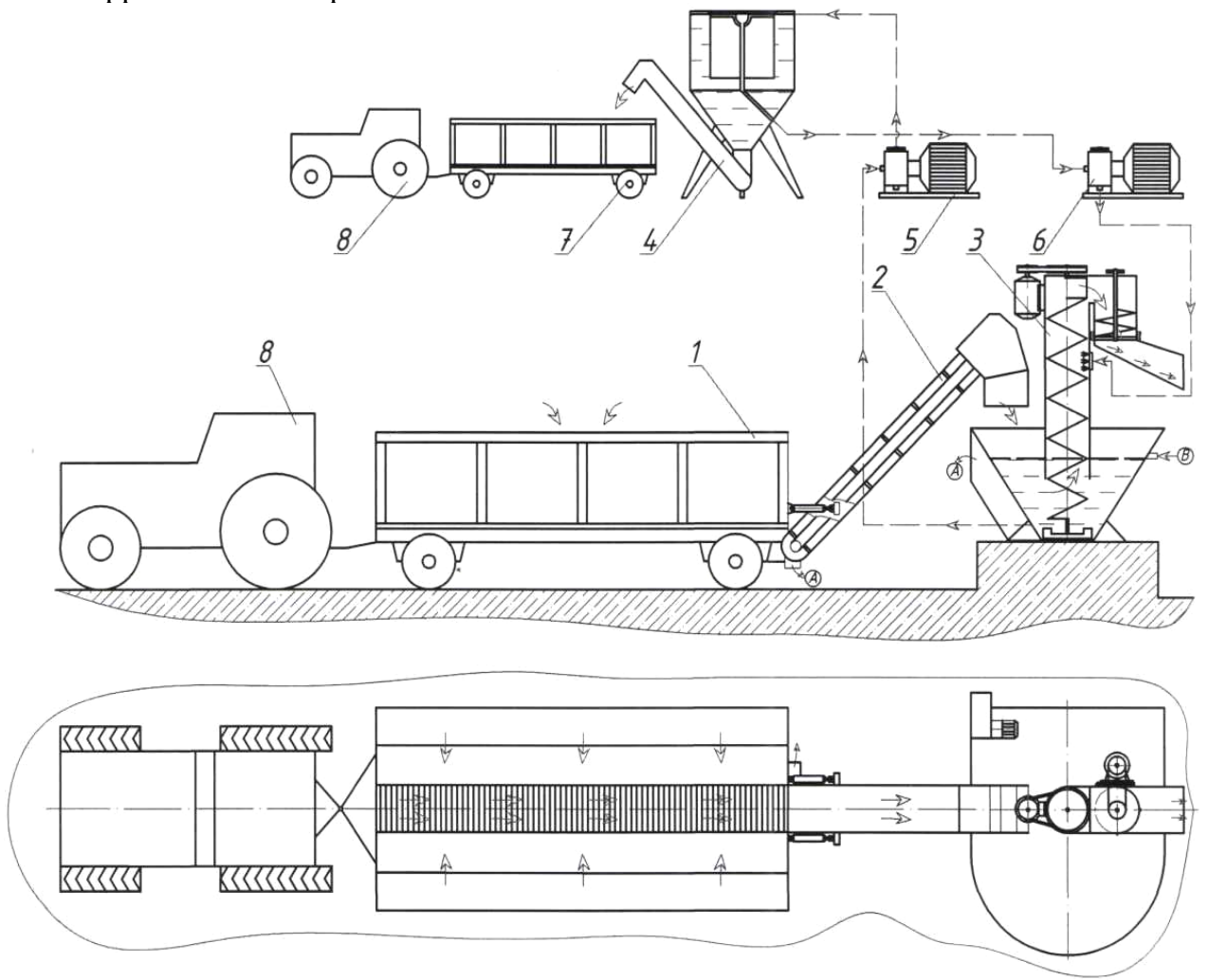

Fig. 1. Technological scheme of BTS delivery and preparation of root crops: 1-feeder-dispenser; 2inclined scraper conveyor; 3-sink-chopper IKM-f-10; 4-sump; 5, 6-pumps; 7-tractor trailer; 8-tractor; a-removal of soil impurities; B-water supply

When considering any production from the standpoint of system analysis, it is possible to distinguish a number of elements, each of which in turn can be considered as a biotechnological system. Each of these elements (subsystems) is characterized by a complex hierarchical structure of relations, to which a systematic approach is also applicable. With regard to the technological process of preparing root crops for feeding, including delivery, washing, grinding and their use in the diet of cows, BTS should be understood as a complex hierarchical structure of orderly interdependent and interrelated devices that ensure the transformation of material (feeding rations) and energy flows in the process of obtaining and processing feed into final products - meat and milk. This relationship should be analyzed using the apparatus of graph theory $[18,19,20]$. On the basis of the technological scheme of BTS we will make the scheme in the form of technological operators (see Fig. 2), the working conditions of which are as follows.

Root crops are loaded into the hopper of the feeder-dispenser 1 and delivered by the tractor 8 to the cooking line. The tractor driver backs up to the sink-chopper 3 and with the help of hydraulic cylinders combines the unloading window of the inclined conveyor 2 with the loading window of the sink-chopper. From the hopper of the feeder-dispenser 1, root 
crops under their own weight are fed to the longitudinal chain-planed conveyor (flow $L_{1}$ ). Due to friction between root crops during their movement along the inclined sides of the side walls of the hopper feeder-dispenser 1 and moving along the scraper conveyor located inside it, there is an initial separation of contaminants (soil impurities, stones and other heavy objects) and their removal (flow $L_{2}$ ). Next, root crops are fed to the inclined scraper conveyor 2 (flow $L_{3}$ ), where also due to friction between themselves and their movement there is an additional separation of contaminants and their removal (flow $L_{4}$ ). Next, the flow of root crops $L_{5}$ enters the sink, where they are finally soaked and washed in the bath from contamination, washed by a stream of purified water (stream $L_{6}$ ), created by the pump from the sump 4. At the same time, the contaminated water (flow $L_{7}$ ) is discharged by the pump into the sump 4 and the stones are removed from the bath by the discharge conveyor of the sink (flow $L_{8}$ ). In the chopper falls stream L5, where the grinding of root crops in accordance with zootechnical requirements. Through the discharge tray of the shredder, root crops enter the hopper of the self-propelled feed feeder-mixer 5 (stream $L_{9}$ ) with an electronic weighing system for all components of the diet and are then issued to agricultural animals.

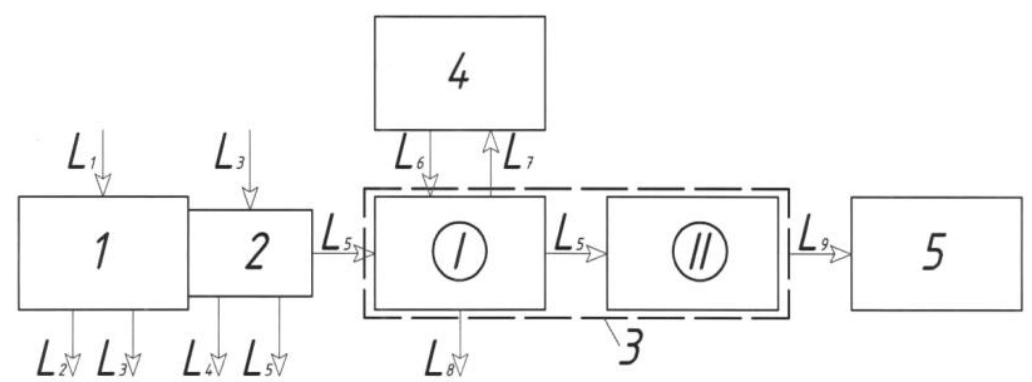

Fig. 2.Operator scheme of delivery and preparation of root crops: 1-feeder-dispenser; 2-inclined scraper conveyor; 3-sink-chopper; 4-sump; 5-feeder-mixer; I-washing bath; II-chopper

Technological flows interact consistently with each other, BTS and the environment, which contributes to the purposeful work of the entire system as a whole. Conditional flows reflect the loss of substance or energy of BTS elements in the environment and various material and energy disturbing effects of the environment on the action of BTS.

Flow characteristics according to the scheme of technological operators are as follows:

$$
\begin{aligned}
& L_{1}=\left\{q_{1} ; W_{1} ; \rho_{1} ; \lambda_{1}\right\} ; L_{2}=\left\{q_{2} ; W_{2} ; \rho_{2} ; \lambda_{2}\right\} ; L_{3}=\left\{q_{3} ; W_{3} ; \rho_{3} ; \lambda_{3}\right\} ; \\
& L_{4}=\left\{q_{4} ; W_{4} ; \rho_{4} ; \lambda_{4}\right\} ; L_{5}=\left\{q_{5} ; W_{5} ; \rho_{5} ; \lambda_{5}\right\} ; L_{6}=\left\{q_{6} ; W_{6} ; \rho_{6} ; \lambda_{6}\right\} ; \\
& L_{7}=\left\{q_{7} ; W_{7} ; \rho_{7} ; \lambda_{7}\right\} ; L_{8}=\left\{q_{8} ; W_{8} ; \rho_{8} ; \lambda_{8}\right\} ; L_{9}=\left\{q_{9} ; W_{9} ; \rho_{9} ; \lambda_{9}\right\},
\end{aligned}
$$

Where $(q)$ - the rate of issuance of root crops;

$(W)$ - moisture content of root crops;

$(p)$ - density of root crops;

$(\lambda)$ - degree of crushing of root crops.

The basis of the BTS analysis is the calculation of material and energy balances in the conditions of the established technological regime. Thus make and solve systems of equations of balances. The system of BTS balance equations is formed by a set of independent equations, including the equations of material balances.

When analyzing the work of BTS consider external sources (root crops), which are supplied to the production line for their preparation, and internal, which display changes in the elements of the system. For each element of BTS on the basis of the laws of conservation of mass and energy, systems of equations of material balances on the total mass flow rate of matter of physical flows are made. This equation has the following form: 


$$
\sum_{j=0}^{n} m_{\mathrm{j}}=0
$$

Equation of material balance on mass consumption of physical and fictitious material flows:

$$
\sum_{j=0}^{n} m_{\mathrm{j}} c_{\mathrm{ji}}+\sum_{n=0}^{k} G_{\mathrm{a}}=0,
$$

where $m_{\mathrm{j}}$ - total mass consumption of the substance;

$c_{\mathrm{ji}}$-the share of the $i$-th element of the component in the unit of total consumption of the substance;

$G_{\mathrm{a}}$ - mass flow rate of $a$-th material dummy flow.

For the $j$-th element of BTS, the linear balance equation of each type of generalized flows is valid:

$$
\sum_{j=0}^{n} Q_{\mathrm{ij}}=0,
$$

where $Q_{\mathrm{ij}}$ - the $i$-th generalized flow associated with elements of the BTS.

The set of equations compiled for all elements of the system form a system of linear equations of balances of one type of generalized BTS flows [12,21]:

$$
[A]+[Q]=0
$$

where $[A]=\left\lfloor a_{\mathrm{ij}}\right\rfloor-$ matrix of a system of equations, elements of which.

For the considered BTS on the basis of the law of conservation of mass it is possible to make nine equations of material balance on mass expenses of streams of processed products (root crops). The resulting system will contain ten equations of material balance with known (free) and unknown (basic) variables. It is quite difficult to choose free variables of the considered BTS equations, as it is necessary to perform a large amount of calculations. The solution of this problem can be greatly simplified if we analyze the topological properties of the BTS. In this case, each system can be put in accordance with the flow graph, which is some topological model of one type of generalized or physical flows of the system. The streaming graphs used for the analysis of technological systems which allow for structural changes for ease of analysis. The complexity of the BTS analysis consists in the large dimension of the problems, as well as in the fact that it has a recycle that makes it difficult to model such a system. Using technological processes in the form of graphs and analysis of the adjacency matrix, it is possible to carry out the decomposition of the system into subsystems of smaller dimension and to investigate the work of the BTS through its subsystems.

Figure 3 shows the flow material graph for the steady-state technological mode of BTS delivery and preparation of root crops. The vertices of the graph on the total mass flow rate of physical flows correspond to the elementsFor the considered BTS on the basis of the law of conservation of mass it is possible to make nine equations of material balance on mass expenses of streams of processed products (root crops). The resulting system will contain ten equations of material balance with known (free) and unknown (basic) variables. It is quite difficult to choose free variables of the considered BTS equations, as it is necessary to perform a large amount of calculations. The solution of this problem can be greatly simplified if we analyze the topological properties of the BTS. In this case, each system can be put in accordance with the flow graph, which is some topological model of one type of generalized or physical flows of the system. The streaming graphs used for the analysis of technological systems which allow for structural changes for ease of analysis. The 
complexity of the BTS analysis consists in the large dimension of the problems, as well as in the fact that it has a recycle that makes it difficult to model such a system. Using technological processes in the form of graphs and analysis of the adjacency matrix, it is possible to carry out the decomposition of the system into subsystems of smaller dimension and to investigate the work of the BTS through its subsystems.

Figure 3 shows the flow material graph for the steady-state technological mode of BTS delivery and preparation of root crops. The vertices of the graph on the total mass flow rate of physical flows correspond to the elementsKroot crop preparation systems that transform the total mass expenditure of physical flows, source $i$ and effluents $S$ of physical flows substances. Arcs meet the mass flow rates of physical flows.

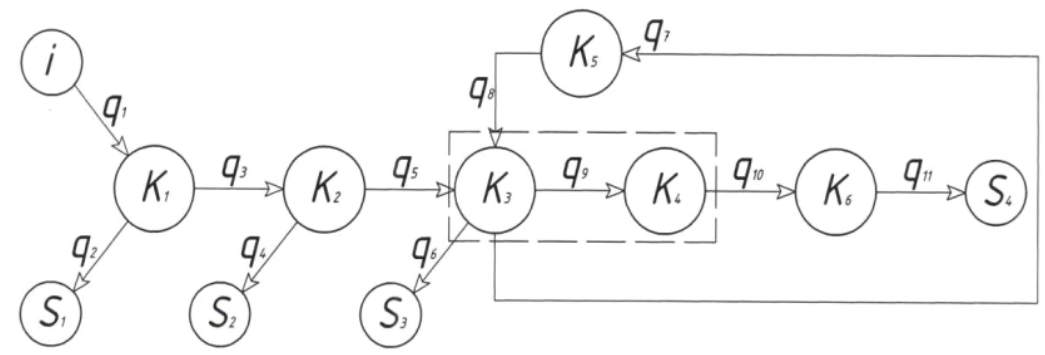

Fig. 3. Flow material graph: $K$-elements; $i$-source; $S$-drains; $q$-flows

By combining all source and sink vertices into one common (zero) vertex, we obtain a cyclic flow graph (figure 4) containing $V=K-1$ vertices and the number of arcs $q$ equal to the number of branches; rank $R=V+1$.

A cyclic flow graph consists of fundamental cycles that include one chord. Selected for the analysis tree of the graph (a formal tree) uniquely determines the edges that are included in each fundamental cycle. The orientation of the fundamental cycle coincides with the direction of the chord that forms it along with the branches of the graph tree.

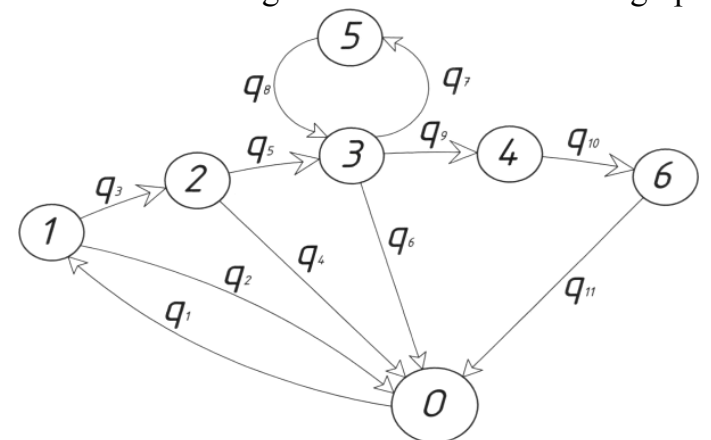

Fig. 4. Cyclic flow graph: 1,2,3,4,5,6 - elements of the BTS system; $q$-flows

For the formal tree (figure 4), the following fundamental cycles can be distinguished:

$$
\mu_{1}=\left\{q_{1}, q_{2}\right\} ; \mu_{2}=\left\{q_{2}, q_{3}, q_{4}\right\} ; \mu_{3}=\left\{q_{4}, q_{6}, q_{5}\right\} ; \mu_{4}=\left\{q_{7}, q_{8}, q_{9}\right\} ; \mu_{5}=\left\{q_{10}, q_{11}\right\} .
$$

In the preparation of the matrix $[C]$ its columns are arranged in ascending order of the chord numbers forming the fundamental cycles, and the rows are arranged in such a way that the rows corresponding to the chords first go, and then the branches of the tree (in ascending order of the numbers) [11]. 
Generally $[C]=[E] /[F]$, where $[E]-$ a unit matrix whose order is $[(e-R)(e-R)] ;[F]-$ matrix of order $R[(e-R)]$; $e-$ number of arcs; $R$ - graph rank.

The complex of equations allows to fully characterize the BTS of delivery, preparation and use of root crops for animal feed.

\section{Results and discussion}

The equations of material balance based on the analysis of the operator scheme of the BTS (figure 2) and the cyclomatic matrix allow to determine the load on all elements included in the BTS. At the same time, it is advisable to choose free variables of the system based on the analysis of its topological properties. Therefore, a consistent study of the nature and strength of these connections allows you to develop and make the most rational decisions in order to improve the efficiency of the use of technology.

Analyzing the flow material graph (figure 3 ) we see that the delivery and preparation of root crops occur in a continuous flow. Losses of root crops mass and their nutritional properties at reduction of technological operations of BTS decrease proportionally to number of elements of system. At the same time, the time of preparation of root crops in the BTS is reduced several times compared to conventional livestock farms and complexes with similar systems. In addition, it allows us to conclude that environmental pollution in the application of BTS is minimized.

\section{Conclusion}

The mathematical models considered in this article allow a reasonable approach to the selection of the main parameters of machines and equipment for the preparation of root crops for animal feed, and in many cases to optimize them. This creates prerequisites for the development of more advanced and productive machines while ensuring the required quality of technological processes with low specific costs and metal consumption.

\section{References}

1. V. Khmelovskyi, S. Rogach, O. Tonkha, Y. Rosamaha, Engineering for Rural Development, 18, 299-304 (2019)

2. V.V. Kafarov, Yu. Naletov, Yu.A. Chernegov, Doklady Chemical technology, 301 (3), 87-90 (2019)

3. A.V. Brusenkov, A.S. Pilyagin, Operation of machinery and equipment for the preparation and distribution of feed in animal husbandry, Theoretical and practical aspects of the development of science and education in the modern world may 16, 5058 (Sofia, Bulgaria, 2017)

4. S.A. Salami, G. Luciano, M.N. O'Grady, J.P. Kerry, A. Priolo, Animal Feed Science and Technology, 251, 37-55 (2019)

5. O.G. Vysotskiy, A.I. Grishenkov, A.M. Khlopyanikov, G.V. Khlopyanikova, A.M. Lobanovsky, Journal of Advanced Research in Dynamical and Control Systems, 11 (2), 412-418 (2019)

6. L. Lalaleo, R. Alcazar, J. Palazon, E. Moyano, R.M. Cusido, M. Bonfill, Journal of Plant Physiology, 228, 197-207 (2018)

7. D. Hidalgo, V. Steinmetz, M. Brossat, L. Tournier-Couturier, R.M. Cusido, P. Corchete, J. Palazon, Engineering in Life Sciences, 17 (4), 413-419 (2017) 
8. K.K.S.A. Albuquerque, W.W.C. Albuquerque, R.M.P.B. Costa, J.M.S. Batista, D.A.V. Marques, R.P. Bezerra, P.N. Herculano, A.L.F Porto, Biocatalysis and Agricultural Biotechnology, 23 (1), 101453 (2020)

9. A. Apazhev, V. Smelik, Y. Shekikhachev, L. Hazhmetov, Engineering for Rural Development, 18, 192-198 (2019)

10. P.Oleinik, N. Cherednichenko, IOP Conference Series: Earth and Environmental Science, 403 (1), 012189 (2019)

11. I. Vorotnikov, M. Gutuev, K. Petrov, O. Esin, Espacios, 38 (23), 22 (2017)

12. V.A. Nemtinov, A.N. Zazulya, V.P. Kapustin, Yu.V. Nemtinov, Journal of Physics: Conf. Series, 1278 (1), 012018 (2019)

13. D.E. Houssaini, S. Khriji,K. Besbes, O. Kanoun, Components and System Design, 323-342 (2018)

14. V.A. Nemtinov, Y.V. Nemtinova, Journal of Computer and Systems Sciences International, 43 (5), 800-804 (2004)

15. O.V. Myalo, S.P. Prokopov, V.V. Myalo, A.S. Soyunov, E.V. Demchuk, IOP Conference Series: Materials Science and Engineering, 582 (1), 012028 (2019)

16. K. Nemtinov, A. Eruslanov, Y. Nemtinova, V. Nemtinov, MATEC Web of Conferences, 224, 02036 (2018)

17. V. Alekseev, D. Lakomov, A. Shishkin, G.Al. Maamari and M. Nasraoui, Jornal of Physics: Conference Series, 1278, 012032 (2019)

18. O. Krol, V. Sokolov, Jornal of Physics: Conference Series, 1084, 012007 (2018)

19. V.A. Nemtinov, Yu.V. Nemtinova, Journal of Computer and Systems Sciences International, 44 (3), 389-398 (2005)

20. V.G. Mokrozub, V.A. Nemtinov A.V. Mokrozub, Chemical and Petroleum Engineering, 53 (5-6), 326-331 (2017)

21. A.B. Borisenko, S.V. Karpushkin, J. of Computer and Systems Sci. Int. 53 (3), 410 419 (2004) 